\title{
Strong potential second Born theory for electron loss to the continuum in collision with heavy targets
}

\author{
D H Jakubassa-Amundsen \\ Physics Section, University of Munich, 85748 Garching, Federal Republic of Germany
}

Received 22 February 1993, in final form 19 May 1993

\begin{abstract}
A second-order Born theory is formulated for the description of forward electron emission from a light projectile in energetic collision with a heavy target atom. This theory is termed "strong potential second Born theory' (SB2) because it accounts for electron propagation in the strong target potential. It is evaluated for the singly inelastic contribution to electron loss, while the first Born approximation is used for the simultaneous projectile-target excitation. From the SB2 theory, the 'impulse approximation' of Hartley and Walters is derived. By comparison with experimental data for the $\mathrm{He}^{+}+\mathrm{Ar}$ system it is shown that both theories account well for the electron intensity, while the asymmetry of the forward peak is only correctly given by SB2. For larger emission angles, consideration of the target potential in the electronic final state is of increasing importance. Then the SB2 becomes inferior to the electron impact approximation.
\end{abstract}

\section{Introduction}

The dominant feature in the momentum distribution of ejected projectile electrons is the electron loss peak which generally appears at electron energies close to $v^{2} / 2$ where $v$ is the collision velocity. For forward electron emission angles, this peak is caused by electrons with low energy in the projectile reference frame (Drepper and Briggs 1976). A special situation arises when the emission angle tends to zero. Then the electron loss peak becomes very narrow and is usually called forward peak or cusp. This cusp is a favourite candidate for testing theories, because its shape is very sensitive to the various theoretical models.

For electrons with a low velocity relative to the projectile, a description in terms of projectile eigenstates is appropriate. The simplest theory is the first-order Born approximation (Drepper and Briggs 1976) which, however, fails to describe the asymmetry of the forward peak (Day 1980). For light targets (e.g. He), the conventional second-order Born theory (which treats the target field perturbatively up to second order) has been found to give a good description of both intensity and shape of the forward peak at intermediate to high impact velocities (Jakubassa-Amudsen 1990).

For heavy targets, however, the first-order Born theory even fails to describe the intensity of the electron loss peak, except at asymptotically large collision velocities (Walters 1975). Even worse, the conventional Born series does not readily converge. The failure of a perturbative expansion in terms of the target field manifests itself in the fact that the second-order Born theory provides electron intensities which considerably exceed those from the first-order Born theory (Kahle 1991), which in turn are already much higher than the experimental intensities. As a consequence, an appropriate description of electron loss in collision with heavy targets must treat the target field non-perturbatively. A theory which includes the target potential to all orders by considering quasielastic scattering of 
the projectile electron from the target (while retaining a projectile final state) has recently been developed by Hartley and Walters (1987). Their 'impulse approximation' gives a good description of the forward peak intensity, but it provides a symmetric cusp like the firstorder approach. This is in contradiction to the experimental data (Man et al 1986, Knudsen et al 1986, Gulyás et al 1989, 1992, Atan et al 1992).

In the present work, the strong potential second Born theory is formulated, which is formally obtained from the conventional second-order Born theory by replacing the electronic propagation in the projectile field by propagation in the target field. Only neutral targets are considered in this work since then special considerations related to the Coulomb interaction are not needed; derivations of higher-order approximations when Coulomb interactions are present asymptotically are discussed by Taulbjerg et al (1990). It is shown that the prescription used by Hartley and Walters (1987) is readily derived from the SB2 theory by means of a full peaking approximation which makes the cusp artificially symmetric.

At larger emission angles, the strong target potential will not only influence the intermediate state, but also the final state of the electron. As a consequence, the strong potential second Born theory will no longer provide an adequate prescription. Instead, electron loss is more readily described as a rearrangement process by means of choosing a final target scattering state for the electron, like in the electron impact approximation (EIA; Jakubassa 1980, Kuzel et al 1992). We shall show the connection between the ElA and the 'impulse approximation' of Hartley and Walters, and evaluate all theories over a range of emission angles. The validity region of any of the theories is estimated from a comparison with experimental data (DuBois and Manson 1990).

It has been demonstrated by Bates and Griffing (1954) that during electron loss, target excitation may take place. Hence, apart from the 'singly inelastic' (SI) process considered so far, where the target remains inert, a second process has to be accounted for, the simultaneous projectile-target excitation, also called 'doubly inelastic' (DI) process. While for electron emission into the backward direction, second Born theories have been formulated for the description of Dl (Wang et al 1992, Kuzel et al 1992), electron loss into the forward direction or total DI loss cross sections are conventionally calculated within the first-order Born approximation (Briggs and Drepper 1978, Day 1981, Hartley and Walters 1987). In the first Born theory, DI is caused by the electron-electron interaction. This interaction is important when there is a small momentum transfer to the projectile electron. This is the case for the electron loss peak region at forward emission angles. Hence, the first Born theory is assumed to give a reasonable estimate for the DI contribution in this region, and will also be used in our calculations.

The paper is organized as follows. In section 2, the SB2 theory for the singly inelastic contribution to electron loss is described, and in section 3, the doubly inelastic contribution is considered. For the $\mathrm{He}^{+}+\mathrm{Ar}$ collision system, the forward peak and its shape parameters are calculated and compared with available experimental data (section 4). Electron loss at larger emission angles is discussed in section 5. Concluding remarks are given in section 6. Atomic units $(\hbar=m=e=1$ ) are used unless otherwise indicated.

\section{The strong potential second Born theory (SB2)}

For the description of projectile ionization in an ion-atom encounter where the target atom remains in its ground state, the so-called elastic or 'singly inelastic' (SI) process, we use the independent electron model. In this model, only the active projectile electron is explicitly 
considered while the target atom and the residual projectile core are represented by effective potentials $V_{\mathrm{T}}$ and $V_{\mathrm{P}}$, respectively. This implies that the electron-electron interaction $V_{\mathrm{ee}}$, which acts simultaneously on two particles, is replaced by its average over the atomic or ionic ground-state configuration.

With this choice of potentials, the exact transition amplitude from a projectile bound state $\psi_{l}^{p}$ to a projectile continuum state $\psi_{f}^{p}$ is given by

$$
a_{f i}^{\mathrm{SI}}=-\mathrm{i} \int \mathrm{d} t\left\langle\psi_{f}^{\mathrm{P}}\left|V_{\mathrm{T}}+V_{\mathrm{T}} G V_{\mathrm{T}}\right| \psi_{i}^{\mathrm{P}}\right\rangle
$$

where $G=\left(\mathrm{i} \partial_{t}-H+\mathrm{i} \varepsilon\right)^{-1}$ is the propagator of the projectile electron which considers the full Hamiltonian $H=T+V_{\mathrm{P}}+V_{\Upsilon}$ with $T$ the kinetic energy of the active electron. The strong potential second Born approximation is obtained by replacing $G$ with the propagator in the strong field, i.e. by neglecting the weaker of the two potentials in $H$. Hence,

$$
\begin{array}{ll}
G \rightarrow G_{\mathrm{T}}=\left(\mathrm{i} \partial_{t}-T-V_{\mathrm{T}}+\mathrm{i} \varepsilon\right)^{-1} & \text { for } Z_{\mathrm{P}}<Z_{\mathrm{T}} \\
G \rightarrow G_{\mathrm{P}}=\left(\mathrm{i} \partial_{t}-T-V_{\mathrm{P}}+\mathrm{i} \varepsilon\right)^{-1} & \text { for } Z_{\mathrm{P}} \geqslant Z_{\Upsilon}
\end{array}
$$

where $Z_{\mathrm{P}}$ and $Z_{\Upsilon}$ are the nuclear charges of projectile and target, respectively. The substitution (2.2b) has been applied for the case of helium targets (Jakubassa-Amundsen 1990). It leads to the conventional second-order Born (B2) theory which is of second order in the perturbing target field $V_{\mathrm{T}}$ while the electron propagates in the field of the parent nucleus, $V_{\mathrm{p}}$.

For heavy targets like Ar (and light projectiles) the substitution (2.2a) has to be made. Inserting further into the transition amplitude (2.1) two complete sets of plane waves $\left|q^{\mathrm{T}}\right\rangle$ and $\left|k^{\mathrm{T}}\right\rangle$ with target-frame momenta $q$ and $k$, respectively, and making the on-shell approximation $\left(1+G_{\mathrm{T}} V_{\mathrm{T}}\right)\left|q^{\mathrm{T}}\right\rangle=\psi_{q}^{\mathrm{T}}$ where $\psi_{q}^{\mathrm{T}}$ is a target scattering eigenstate with momentum $q$, one obtains

$$
a_{f i}^{\mathrm{SB} 2}=-\mathrm{i} \int \mathrm{d} t \int \mathrm{d} k \mathrm{~d} q\left\langle\psi_{f}^{\mathrm{P}} \mid k^{\mathrm{T}}\right\rangle\left\langle k^{\mathrm{T}}\left|V_{\mathrm{T}}\right| \psi_{q}^{\mathrm{T}}\right\rangle\left\langle\boldsymbol{q}^{\mathrm{T}} \mid \psi_{l}^{\mathrm{P}}\right\rangle
$$

For energetic collisions, a straight-line path, $R=b+v t$, can be used for the internuclear motion. In this expression, $\boldsymbol{R}$ is the internuclear coordinate, $\boldsymbol{b}$ the impact parameter and $v$ the collision velocity. Introducing the Fourier transforms $\varphi_{i}^{\mathrm{P}}$ and $\varphi_{f}^{\mathrm{P}}$ of initial and final states, respectively, and defining the generalized scattering amplitude $f$ by the relation

$$
\left\langle k^{\mathrm{T}}\left|V_{\mathrm{T}}\right| \psi_{q}^{\mathrm{T}}\right\rangle=\left\langle\psi_{k}^{\mathrm{T}}\left|V_{\mathrm{T}}\right| q^{\mathrm{T}}\right\rangle=-\frac{1}{(2 \pi)^{2}} f(q, k)
$$

(2.3) is written in the following way

$a_{f i}^{\mathrm{SB2}}=\frac{\mathrm{i}}{2 \pi} \int \mathrm{d} q^{\prime} \mathrm{e}^{-\mathrm{i} q^{\prime} b} \delta\left(\Delta \varepsilon_{f i}^{\mathrm{P}}-q^{\prime} v\right) \int \mathrm{d} k \varphi_{f}^{\mathrm{P} *}(k-v) \varphi_{i}^{\mathrm{P}}\left(q^{\prime}+k-v\right) f\left(q^{\prime}+k, k\right)$

where the substitution $q^{\prime}=q-k$ has been made, and $\Delta \varepsilon_{f i}^{\mathrm{P}}=\varepsilon_{f}^{\mathrm{P}}-\varepsilon_{i}^{\mathrm{P}}$ is the excitation energy of the projectile electron.

Equation (2.5) has a simple intuitive interpretation. For fast collisions, the projectile electron behaves like a quasifree particle which scatters elastically on the target. The initial and final momenta of the electron are distributed according to the respective momentum 
space wavefunctions $\varphi_{i}^{\mathrm{P}}$ and $\varphi_{f}^{\mathrm{P}}$. The shift of the momenta $k$ and $q^{\prime}+k$ by the velocity $v$ in these functions accounts for the relative motion between the projectile and the target reference frames. This intuitive picture of electron loss induced by a heavy target was taken by Hartley and Walters (1987) as the starting point of their theory. In deducing their final result, they made use of the fact that for light projectiles, the momentum space wavefunction $\varphi_{f}^{\mathrm{P}}(k-v)$ is strongly peaked at $k=v+\kappa_{f}=k_{f}$, where $k_{f}$ and $\kappa_{f}$ are the final electronic momenta in the target and projectile frame, respectively. Consequently, a full peaking approximation was applied to the scattering amplitude $f\left(q^{\prime}+k, k\right)$ in $(2.5)$ by taking it outside the $k$-integral at the fixed momentum $k=k_{f}$. The remaining integral is easily evaluated, yielding just the ionization matrix element for the projectile electron. Hence, the peaked SB2 theory leads to the following result applied by Hartley and Walters

$$
a_{f i}^{\mathrm{pSB2}}=\frac{\mathrm{i}}{2 \pi} \int \mathrm{d} q^{\prime} \mathrm{e}^{-\mathrm{i} q^{\prime} b} \delta\left(\Delta \varepsilon_{f i}^{\mathrm{P}}-q^{\prime} v\right) f\left(q^{\prime}+k_{f}, k_{f}\right)\left\langle\psi_{f}^{\mathrm{P}}\left|\mathrm{e}^{-\mathrm{i} q^{\prime} r_{\mathrm{p}}}\right| \psi_{i}^{\mathrm{P}}\right\rangle
$$

where $r_{P}$ is the projectile-frame electron coordinate. This equation has an intriguing similarity with the first-order Born theory. The latter is obtained by simply replacing the exact target scattering amplitude with its first-order approximation, i.e. with the Fourier transformed target field $\tilde{V}_{\mathrm{T}}$

$$
f\left(q^{\prime}+k_{f}, k_{f}\right) \rightarrow-(2 \pi)^{1 / 2} \tilde{V}_{\mathrm{T}}\left(-q^{\prime}\right)
$$

where $q^{\prime}$ is the momentum transfer.

The differential cross section for the emission of electrons with energy $E_{f}=k_{f}^{2} / 2$ into the solid angle $\mathrm{d} \Omega_{f}$ is obtained by means of integrating the transition probability over all impact parameters

$$
\frac{\mathrm{d}^{2} \sigma^{\mathrm{Sl}}}{\mathrm{d} E_{f} \mathrm{~d} \Omega_{f}}=k_{f} \int \mathrm{d} b\left|a_{f i}^{\mathrm{SI}}\right|^{2}=\frac{k_{f}}{v} \int \mathrm{d} q^{\prime} \delta\left(\Delta \varepsilon_{f i}^{\mathrm{P}}-q^{\prime} v\right)\left|M_{f i}^{\mathrm{SI}}\left(q^{\prime}\right)\right|^{2}
$$

where $M_{f i}^{S 1}\left(q^{\prime}\right)$ denotes the integrand to the right of the $\delta$-function in (2.5) or (2.6). In the peaked SB2 case, one has

$$
M_{f i}^{\mathrm{pSB} 2}\left(q^{\prime}\right)=f\left(q^{\prime}+k_{f}, k_{f}\right)\left\langle\psi_{f}^{\mathrm{P}}\left|\mathrm{e}^{-\mathrm{i} q^{\prime} r_{\mathrm{p}}}\right| \psi_{i}^{\mathrm{P}}\right\rangle
$$

From (2.8) with (2.9) it is readily seen that the 'impulse approximation' of Hartley and Walters (in our nomenclature, the peaked strong potential second Born theory) shows a cusp structure which is very much like the one obtained from the first-order Born approximation. As has been shown previously (Jakubassa-Amundsen 1990), the asymmetry of the cusp can be related to a phase factor in the momentum space projectile state $\varphi_{f}^{\mathrm{P}}$ or equivalently, in the projectile ionization matrix element $\left\langle\psi_{f}^{\mathrm{P}}\left|\exp \left(-\mathrm{i} q^{\prime} r_{\mathrm{p}}\right)\right| \psi_{1}^{\mathrm{P}}\right\rangle$. This phase gets lost in the differential cross section (2.8) where only the squared modulus of the matrix element is entering. The other factor in the integrand, the target scattering amplitude, is a smoothly varying function in the cusp region and therefore only affects the peak intensity, but not the shape. Hence, the resulting cusp is symmetric.

In order to retain the cusp asymmetry, the $\mathrm{SB} 2$ transition matrix element

$$
M_{f i}^{\mathrm{SB} 2}\left(q^{\prime}\right)=\int \mathrm{d} k \varphi_{f}^{\mathrm{P} *}(k-v) \varphi_{i}^{\mathrm{P}}\left(q^{\prime}+k-v\right) f\left(q^{\prime}+k, k\right)
$$


has to be evaluated without such a peaking approximation. In the following, we restrict ourselves to one-electron projectiles. In (2.10), we make a coordinate transformation $k^{\prime}=k-k_{f}$ and choose $\kappa_{f}=k_{f}-v$ as quantization axis for the spherical coordinates of $k^{\prime}$. This is necessary to cope efficiently with the pole structure of the Fourier transformed Coulomb wave $\varphi_{f}^{\text {P }}$

$\varphi_{\kappa f}^{\mathrm{P} *}\left(k^{\prime}+\kappa_{f}\right)=\frac{Z_{\mathrm{P}}}{\pi^{2}} \mathrm{e}^{\pi \eta / 2} \Gamma(1-\mathrm{i} \eta) \frac{\left(k^{\prime}+2 \kappa_{f} \cos \vartheta_{k^{\prime}, \kappa_{f}}-\mathrm{i} \varepsilon\right)^{-\mathrm{i} \eta-1}}{k^{\prime 3-\mathrm{i} \eta}} \quad \eta=Z_{\mathrm{P}} / \kappa_{f}, \varepsilon \rightarrow 0$.

For the scattering amplitude $f\left(q^{\prime}+k^{\prime}+k_{f}, k^{\prime}+k_{f}\right)$ we use the on-shell approximation of Hartley and Walters (1987)

$$
f\left(k_{1}, k_{2}\right)=f_{\mathrm{e}}(K, \theta) \quad K=\max \left(k_{1}, k_{2}\right) \quad \sin \theta / 2=\left|k_{1}-k_{2}\right| /(2 K)
$$

where $f_{\mathrm{e}}(K, \theta)$ is the amplitude for elastic scattering of an electron (with momentum $K$ and scattering angle $\theta$ ) on the target potential. In the electron loss peak region, the main contributions come from momenta near $k_{1}=k_{2}$ where (2.12) is exact. In the rare cases where $\left|k_{1}-k_{2}\right| / 2 K$ exceeds one, $K$ is taken to be $\left|k_{1}-k_{2}\right| / 2$ such that $\sin \theta / 2=1$.

We introduce one minor approximation into the evaluation of $M_{f i}^{\mathrm{SB2}}$ by neglecting the variation of the scattering amplitude with the azimuthal angle $\varphi_{k^{\prime}}$. The use of a fixed value $\bar{\varphi}_{k^{\prime}}$ for this angle may be justifed from the fact that the initial state function $\varphi_{i}^{\mathrm{P}}\left(q^{\prime}+k^{\prime}+\kappa_{f}\right)$ is strongly peaked at $\left(q^{\prime}+k^{\prime}+\kappa_{f}\right)^{2}=0$ for light projectiles. We determine $\cos \left(\bar{\varphi}_{k^{\prime}}-\varphi_{q^{\prime}}\right)$ (where $\varphi_{q}$ is the azimuthal angle of $q^{\prime}$ ) from this relation as long as we get a value in the interval $(-1,1)$. For values $>1$ or $<-1$, we set $\cos \left(\bar{\varphi}_{k^{\prime}}-\varphi_{q^{\prime}}\right)=1$ or -1 , respectively. This gives

$$
\begin{aligned}
& \bar{\varphi}_{k^{\prime}}= \begin{cases}\varphi_{q^{\prime}}+\cos ^{-1}\left(-C_{0} / B\right) & \text { for }\left|C_{0} / B\right| \leqslant 1 \\
\varphi_{q^{\prime}}+\pi & \text { for }\left|C_{0} / B\right|>1 \text { and } C_{0}>0 \\
\varphi_{q^{\prime}} & \text { for }\left|C_{0} / B\right|>1 \text { and } C_{0}<0\end{cases} \\
& C_{0}=q^{\prime 2}+k^{\prime 2}+\kappa_{f}^{2}+2 k^{\prime} k_{f} \cos \vartheta_{k^{\prime}, \kappa_{f}}+2 q^{\prime} \cos \vartheta_{q^{\prime}, \kappa_{f}}\left(\kappa_{f}+k^{\prime} \cos \vartheta_{k^{\prime}, \kappa_{f}}\right) \\
& B=2 q^{\prime} k^{\prime} \sin \vartheta_{q^{\prime}, \kappa_{f}} \sin \vartheta_{k^{\prime}, \kappa_{f}} .
\end{aligned}
$$

Even with this approximation, the scattering amplitude is subject to a fourfold integration in $(2.8)$ which smoothens the functional dependence of $f_{\mathrm{e}}$. Hence, we do not expect the above approximation to be of much significance. With $\varphi_{k^{\prime}}=\bar{\varphi}_{k^{\prime}}$ in $f_{e}$, the integration over $\varphi_{k^{\prime}}$ affects only $\varphi_{l}^{\mathrm{P}}$ and is easily carried out for a hydrogenic 1s state

$$
\begin{aligned}
\int_{0}^{2 \pi} \mathrm{d} \varphi_{k^{\prime}} \varphi_{i}^{\mathrm{P}}\left(q^{\prime}+k^{\prime}+\kappa_{f}\right) & =\frac{2 \sqrt{2}}{\pi} Z_{\mathrm{P}}^{5 / 2} \int_{0}^{2 \pi} \mathrm{d} \varphi_{k^{\prime}} \frac{1}{\left(A+B \cos \varphi_{k^{\prime}}\right)^{2}} \\
& =4 \sqrt{2} Z_{\mathrm{P}}^{5 / 2} \frac{A}{\left(A^{2}-B^{2}\right)^{3 / 2}}
\end{aligned}
$$

The SB2 approximation for the doubly differential electron loss cross section is thus calculated from (2.8) with (2.10)-(2.14):

$$
\begin{aligned}
\frac{\mathrm{d}^{2} \sigma^{\mathrm{SB} 2}}{\mathrm{~d} E_{f} \mathrm{~d} \Omega_{f}}= & \frac{64 Z_{\mathrm{p}}^{7} k_{f}}{\pi^{3} v^{2}} \frac{\eta}{1-\mathrm{e}^{-2 \pi \eta}} \int_{q_{\mathrm{min}}}^{\infty} q^{\prime} \mathrm{d} q^{\prime} \int_{0}^{2 \pi} \mathrm{d} \varphi_{q^{\prime}} \\
& \times\left|\int_{0}^{\infty} \frac{\mathrm{d} k^{\prime}}{k^{\prime \mathrm{i}-\mathrm{i} \eta}} \int_{-1}^{1} \mathrm{~d} x\left(k^{\prime}+2 \kappa_{f} x-\mathrm{i} \varepsilon\right)^{-\mathrm{i} \eta-1} \frac{A}{\left(A^{2}-B^{2}\right)^{3 / 2}} f_{\mathrm{e}}(K, \theta)\right|^{2}
\end{aligned}
$$


with $x=\cos \vartheta_{k^{\prime}, \kappa_{f}}, q_{\min }=\Delta \varepsilon_{f i}^{\mathrm{P}} / v$ and $\cos \vartheta_{q^{\prime}}=q_{\min } / q^{\prime}$ where $v$ has been chosen as the quantization axis for $\boldsymbol{q}^{\prime}$. Momentum and scattering angle in $f_{\mathrm{e}}(K, \theta)$ are calculated according to (2.12) from $k_{1}=q^{\prime}+k^{\prime}+k_{f}$ and $k_{2}=k^{\prime}+k_{f}$ with the substitution $\varphi_{k^{\prime}} \rightarrow \bar{\varphi}_{k^{\prime}}$ from (2.13). Further numerical details are deferred to the appendix.

\section{The inelastic contribution to forward electron emission}

We now consider electron loss in the case where the final state $\phi_{f}^{\mathrm{T}}$ of the target electrons differs from the initial ground state $\phi_{i}^{\mathrm{T}}$. Two processes can be distinguished in this inelastic contribution (also termed 'doubly inelastic (DI) contribution) to electron loss. One is the 'correlated' DI process, which concerns the simultaneous excitation of a target and a projectile electron by the electron-electron interaction $V_{\mathrm{ee}}$. The other process involves two successive electron-nucleus couplings. In this 'uncorrelated' Dl process, the projectile electron is ejected by the target field $V_{\Upsilon}$ and the target electron by the interaction with the projectile core, $V_{\mathrm{Pe}}$. The DI transition amplitude is the sum of the correlated and the uncorrelated term

$$
\begin{aligned}
& a_{f i}^{\mathrm{DI}}=a_{f i}^{\mathrm{cor}}+a_{f i}^{\mathrm{unc}} \\
& a_{f i}^{\mathrm{cor}}=-\mathrm{i} \int \mathrm{d} t\left\langle\phi_{f}^{\mathrm{T}} \psi_{f}^{\mathrm{P}}\left|V_{\mathrm{ee}}+\hat{V}_{\mathrm{ee}} \tilde{G} V_{\mathrm{T}}+V_{\mathrm{T}} \tilde{G} \hat{V}_{\mathrm{ee}}\right| \psi_{i}^{\mathrm{P}} \phi_{i}^{\mathrm{T}}\right\rangle \\
& a_{f i}^{\mathrm{unc}}=-\mathrm{i} \int \mathrm{d} t\left\langle\phi_{f}^{\mathrm{T}} \psi_{f}^{\mathrm{P}}\left|V_{\mathrm{Pe}} \tilde{G} V_{\mathrm{T}}+V_{\mathrm{T}} \tilde{G} V_{\mathrm{Pe}}\right| \psi_{i}^{\mathrm{P}} \phi_{i}^{\mathrm{T}}\right\rangle
\end{aligned}
$$

where $\hat{V}_{\mathrm{ee}}=V_{\mathrm{ee}}-\left\langle\phi_{i}^{\mathrm{T}}\left|V_{\mathrm{ee}}\right| \phi_{i}^{\mathrm{T}}\right\rangle$ is the two-body interaction not considered in $V_{\mathrm{T}}$, and $\tilde{G}=\left(\mathrm{i} \partial_{t}-H_{\mathrm{T}}-T-V_{\mathrm{P}}-V_{\mathrm{T}}-V_{\mathrm{pe}}+\mathrm{i} \varepsilon\right)^{-1}$ is the propagator of the total projectiletarget system in the independent particle approximation, with $H_{\mathrm{T}}$ the Hamiltonian of the unperturbed target electrons. Terms which are of higher than first order in $\hat{V}_{\mathrm{ee}}$ have been omitted, since their contribution is expected to be small. Likewise, terms $\sim \hat{V}_{e e} \tilde{G} V_{\mathrm{Pe}}$ are neglected as compared to the terms $\sim V_{\mathrm{T}} \tilde{G} V_{\mathrm{pe}}$, and also terms where the potentials act only on the same electron (like $V_{\mathrm{T}} \tilde{G} V_{\mathrm{T}}$ or $V_{\mathrm{Pe}} \tilde{G} V_{\mathrm{Pe}}$ ) have been discarded. In the strong potential second Born approximation, the projectile electron propagates in the stronger of the fields $\left(V_{\mathrm{P}}, V_{\mathrm{T}}\right)$ while target excitation is treated to first order. Hence, SB2 consists in the approximation

$$
\tilde{G} \rightarrow \begin{cases}\tilde{G}_{\mathrm{T}}=\left(\mathrm{i} \partial_{t}-H_{\mathrm{T}}-T-V_{\mathrm{T}}+\mathrm{i} \varepsilon\right)^{-1} & Z_{\mathrm{P}}<Z_{\mathrm{T}} \\ \tilde{G}_{\mathrm{P}}=\left(\mathrm{i} \partial_{t}-H_{\Upsilon}-T-V_{\mathrm{P}}+\mathrm{i} \varepsilon\right)^{-1} & Z_{\mathrm{P}} \geqslant Z_{\mathrm{T}}\end{cases}
$$

For a helium target, the uncorrelated contribution of (3.1) with the substitution (3.2b) plus the first-order correlated contribution to electron loss have been evaluated previously (Jakubassan Amundsen 1990). The correlated contribution up to second order has recently been considered by Wang et al (1992), however, with the replacement $\bar{G} \rightarrow\left(\mathrm{i} \partial_{l}-H_{\mathrm{T}}-T+\mathrm{i} \varepsilon\right)^{-\mathrm{I}}$ which corresponds to a field-free propagation of the projectile electron. This approximation is doubtful in the case of heavy targets.

Let us consider the heavy-target case, (3.1) with (3.2a), in somewhat more detail. The dependence of the propagator $\tilde{G}_{T}$ on the target electrons is readily eliminated upon inserting a complete set of eigenstates to $\tilde{G}_{\mathrm{T}},\left|\phi_{n}^{\mathrm{T}} * \psi_{n^{\prime}}^{\mathrm{T}}\right\rangle$, into each of the second-order terms such 
that $\tilde{G}_{\mathrm{T}}$ can be reduced to an energy denominator. Due to the orthonormality of the target eigenstates $\phi_{n}^{\mathrm{T}}, \phi_{t}^{\mathrm{T}}$ and $\phi_{f}^{\mathrm{T}}$, one is left with

$$
\begin{aligned}
& a_{f i}^{\mathrm{DI}, \mathrm{SB} 2}=-\mathrm{i} \int \mathrm{d} t\left[\left\langle\psi_{f}^{\mathrm{P}}\left|U_{f i}^{\mathrm{T}}\right| \psi_{i}^{\mathrm{P}}\right\rangle+\left\langle\psi_{f}^{\mathrm{P}}\left|U_{f i}^{\mathrm{T}} G_{\mathrm{T}} V_{\mathrm{T}}+V_{\mathrm{T}} G_{\mathrm{T}} U_{f i}^{\mathrm{T}}\right| \psi_{i}^{\mathrm{P}}\right\rangle\right] \\
& -\mathrm{i} \int \mathrm{d} t\left\langle\phi_{f}^{\mathrm{T}}\left|V_{\mathrm{Pe}}\right| \phi_{i}^{\mathrm{T}}\right\rangle\left\langle\psi_{f}^{\mathrm{P}}\left|G_{\mathrm{T}} V_{\mathrm{T}}+V_{\mathrm{T}} G_{\mathrm{T}}\right| \psi_{i}^{\mathrm{P}}\right\rangle \\
& U_{f i}^{\mathrm{T}}=\left\langle\phi_{f}^{\mathrm{T}}\left|V_{\mathrm{ee}}\right| \phi_{i}^{\mathrm{T}}\right\rangle
\end{aligned}
$$

where the first time integral is the correlated and the second integral the uncorrelated term. The orthogonality between $\phi_{i}^{\mathrm{T}}$ and $\phi_{f}^{\mathrm{T}}$ allows for a replacement of $\hat{V}_{\mathrm{ee}}$ by $V_{\mathrm{ee}}$. We have reintroduced the propagator of the projectile electron, $G_{\mathrm{T}}=\left(\mathrm{i} \partial_{t}-T-V_{\mathrm{T}}+\mathrm{i} \varepsilon\right)^{-1}$. As a consequence of the presence of $G_{T}$ in the projectile electron transition matrix elements, overlap terms between projectile states and the target eigenstates $\psi_{n^{\prime}}^{\mathrm{T}}$ will occur. Hence, the evaluation of (3.3) is far more intricate than in the case of light targets.

Following the line of earlier investigations of forward electron ejection by heavy targets (e.g. Hartley and Walters 1987, DuBois and Manson 1990), we restrict ourselves to the first-order Born term (B1) of the DI contribution,

$$
a_{f i}^{\mathrm{DI}, \mathrm{B} 1}=-\mathrm{i} \int \mathrm{d} t\left\langle\psi_{f}^{\mathrm{P}}\left|U_{f i}^{\mathrm{T}}\right| \psi_{i}^{\mathrm{P}}\right\rangle
$$

For the evaluation of the electron loss cross section, one has to sum over the excited target states. Conventionally, this sum is evaluated with the help of a closure approximation, after replacing the target excitation energy by an average value $\overline{\Delta E}_{f i}$. According to the prescription suggested by Day (1981), we take $\overline{\Delta E}_{f i}=I^{\mathrm{T}}+\kappa_{f}^{2} / 2$ where $I^{\mathrm{T}}$ is the ionization potential of the target. The resulting doubly differential cross section is given by (see e.g. Jakubassa-Amundsen 1990)

$$
\frac{\mathrm{d}^{2} \sigma^{\mathrm{DI} \mathrm{BI}}}{\mathrm{d} E_{f} \mathrm{~d} \Omega_{f}}=\frac{8 k_{f}}{v^{2}} \int_{\bar{q}_{\mathrm{man}}}^{\infty} \frac{\mathrm{d} q}{q^{3}} S_{\mathrm{in}}(q) \int_{0}^{\pi} \mathrm{d} \varphi_{q}\left|\left\langle\psi_{f}^{\mathrm{P}}\left|\mathrm{e}^{\mathrm{i} q r_{\mathrm{P}}}\right| \psi_{i}^{\mathrm{P}}\right\rangle\right|^{2}
$$

where $\bar{q}_{\min }=\left(\Delta \varepsilon_{f i}^{\mathrm{P}}+\overrightarrow{\Delta E}_{f i}\right) / v$ and $S_{\text {in }}$ is the incoherent scattering form factor.

\section{The forward peak in comparison with experiment}

At the cusp maximum, $k_{f}=v$ (i.e. $\kappa_{f}=0$ ), the doubly differential cross section for electron loss to the continuum is divergent as a consequence of the normalization constant of the projectile Coulomb wave $\psi_{f}^{\mathrm{P}}$. Hence, the measured electron intensities depend strongly on the resolution of the spectrometer. In order to compare theory with experiment, the cross section has to be averaged over the detector resolution. Since in most experiments, the energy resolution is considerably better than the angular resolution $\theta_{0}$, it is in general sufficient to average over $\theta_{0}$ only. Taking SI as well as DI contributions into consideration, the electron intensity is determined from

$$
\left(\frac{\mathrm{d}^{2} \sigma}{\mathrm{d} E_{f} \mathrm{~d} \Omega_{f}}\right)_{\theta_{0}}=\frac{1}{1-\cos \theta_{0}} \int_{0}^{\theta_{0}} \sin \vartheta_{f} \mathrm{~d} \vartheta_{f}\left(\frac{\mathrm{d}^{2} \sigma^{\mathrm{SI}}}{\mathrm{d} E_{f} \mathrm{~d} \Omega_{f}}+\frac{\mathrm{d}^{2} \sigma^{\mathrm{DI}}}{\mathrm{d} E_{f} \mathrm{~d} \Omega_{f}}\right) .
$$


In order to compare experiments performed with different detector systems, cusp shape parameters are commonly defined which are independent of $\theta_{0}$ or which obey simple scaling relations. In contrast to electron capture to continuum, it is possible for electron loss to define $\theta_{0}$-independent parameters. For this aim, the doubly differential cross section is transformed into the projectile frame and expanded in terms of $\kappa_{f}$ and Legendre polynomials $P_{l}\left(\cos \vartheta_{f}^{\prime}\right)$, where $\vartheta_{f}^{\prime}$ is the projectile-frame electron emission angle (Meckbach et al 1981)

$$
\frac{\mathrm{d}^{2} \sigma}{\mathrm{d} E_{f} \mathrm{~d} \Omega_{f}}=\frac{k_{f}}{\kappa_{f}} \frac{\mathrm{d}^{2} \sigma}{\mathrm{d} \varepsilon_{f} \mathrm{~d} \Omega_{f}^{\prime}}=\frac{k_{f}}{\kappa_{f}} \sum_{n, l=0}^{\infty} B_{n l} K_{f}^{n} P_{l}\left(\cos \vartheta_{f}^{\prime}\right)
$$

with $\varepsilon_{f}=\kappa_{f}^{2} / 2$. Since the cross section is an analytic function of $\vartheta_{f}^{\prime}$ even at $\kappa_{f}=0$, the expansion (4.2) readily converges. The coefficients $B_{n l}$ are constants which are not affected when the average over the detector resolution is performed, and therefore can be used to define the cusp properties. Theoretically, the $B_{n l}$ are calculated from an inversion of (4.2). Near the cusp maximum, i.e. for $n=0$, one has

$$
B_{0 l}=\frac{2 l+1}{2} \int_{-1}^{1} \mathrm{~d}\left(\cos \vartheta_{f}^{\prime}\right) P_{l}\left(\cos \vartheta_{f}^{\prime}\right)\left[\frac{k_{f}}{k_{f}} \frac{\mathrm{d}^{2} \sigma}{\mathrm{d} E_{f} \mathrm{~d} \Omega_{f}}\right]_{k_{f}=0} .
$$

For an experimental determination of the shape parameters $B_{n l}$, the series (4.2) is truncated to six terms (or less) and folded with the spectrometer function. The $B_{n l}$ are then obtained from a fit to the measured spectra.

The parameter $B_{00}$ is related to the intensity of the cusp. For small angles $\vartheta_{f}, \theta_{0}$ (up to a few degrees) one has to a good approximation

$$
\begin{aligned}
& \left.\frac{\mathrm{d}^{2} \sigma}{\mathrm{d} E_{f} \mathrm{~d} \Omega_{f}}\left(\vartheta_{f}, k_{f}\right)\right|_{\max }=\frac{1}{\vartheta_{f}} B_{00} \\
& \left.\left\langle\frac{\mathrm{d}^{2} \sigma}{\mathrm{d} E_{f} \mathrm{~d} \Omega_{f}}\right\rangle_{\theta_{0}}\right|_{\max }=\frac{2}{\theta_{0}} B_{00}
\end{aligned}
$$

where 'max' indicates the cusp maximum, and the angles are measured in radians. The parameters $B_{00}$ for the $\mathrm{He}^{+}+\mathrm{Ar}$ system are shown in figure 1 as a function of the collision velocity. Calculations have been performed in the first Born approximation, the conventional second Born (B2) approximation, as well as in the peaked and unpeaked SB2 theory (using (2.8) with (2.9), and (2.15), respectively) with the first-order DI contribution from (3.5). In the latter case, for numerical reasons the shape parameters were evaluated at $k_{f}=0.1$ instead of $\kappa_{f}=0$. This does not introduce any significant error, since the projectileframe cross sections depend only weakly on $\kappa_{f}$ for $\kappa_{f} \lesssim 0.2$. Comparison is made with the experimental data from Knudsen et al (1986), Köver et al (1989) and Atan et al (1990). The datum point at the lowest velocity has been extracted from the experimental cusp maximum with the help of $(4.4 b)$. While the first-order Born approximation, and even more the B2 theory strongly overestimate the data, both the peaked and unpeaked SB2 results are in reasonable agreement with experiment except for the highest velocities. The SB2 theory for the singly inelastic contribution is indeed applicable down to very low impact velocities (for strongly asymmetric collision systems) because the strong field is included to all orders. In contrast, the first Born approximation for the doubly inelastic contribution breaks down near $v \sim 1$, which in turn will lead to unreliable values of the shape parameters. This 
can be understood from the presence of a threshold effect for the simultaneous projectiletarget excitation by $V_{\text {ee }}$. The corresponding cross section will rapidly decrease when the mean electron impact energy $v^{2} / 2$ falls below the projectile excitation energy, since a large momentum transfer is required in that case. As has been pointed out by Montenegro and Meyerhof (1991), the applied closure approximation will also be incorrect at such low collision velocities. A correct description of the DI contribution at $v \lesssim 1$ should therefore improve on the closure approximation, but more importantly, should include the uncorrelated DI process since projectile-target excitation by the electron-nucleus interaction is expected to be strongly dominating.

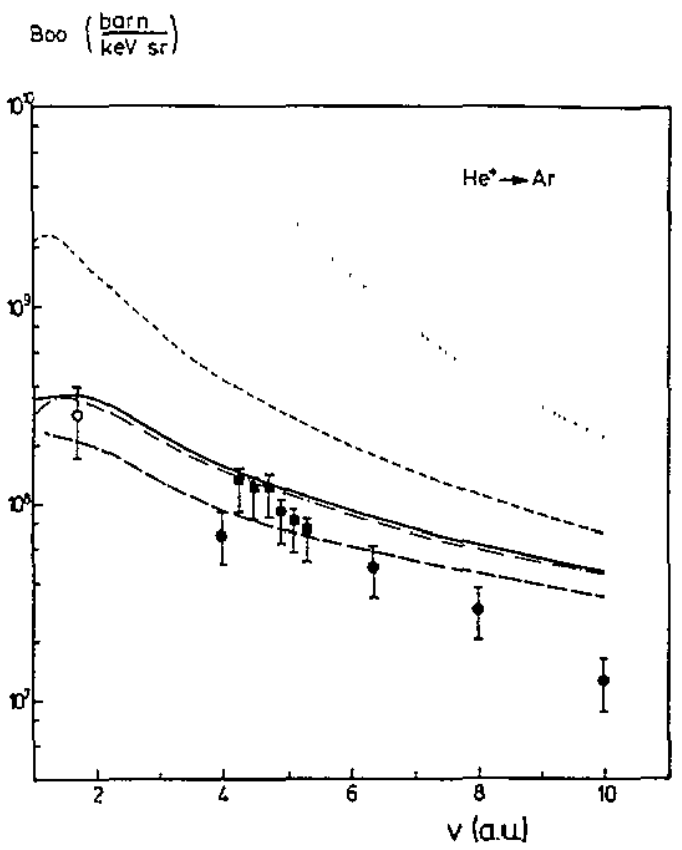

Figure 1. Cusp intensity parameter $B_{00}$ for electron loss (ELC) in $\mathrm{He}^{+}+\mathrm{Ar}$ collision as a function of impact velocity. Theory: SB2 (-), peaked SB2 (- , - ), first Born approximation (- - -), second Bom approximation $\mathrm{B} 2(\ldots$.$) , all theories with SI and DI contributions included; 5B2$ (_ _ - ) with s! only. Experiment: - Knudsen et al (1986) and Jensen (1986), coincidence data; E, Atan et al (1990), singles data; J, Kövér et al (1989), coincidence data.

Figure 2 depicts the ratio $\beta_{1}=B_{01} / B_{00}$ which determines the skewness of the forward peak. A higher intensity on the low-energy wing of the peak corresponds to a negative value of $\beta_{1}$. Such negative $\beta_{1}$ are predicted by the (unpeaked) SB2 theory, whereas the peaked SB2 as well as the first-order Bom theory give $\beta_{1} \approx 0$. Apart from the theories for $\mathrm{He}^{+}+\mathrm{Ar}$, we have included in figure 2 the $\mathrm{B} 2$ result for $\mathrm{He}^{+}+\mathrm{He}$ taken from Jakubassa-Amundsen (1990), in order to show the target dependence of $\beta_{1}$. The increase of $-\beta_{1}$ from $\mathrm{He}$ to $\mathrm{Ar}$ at fixed collision velocity is in quantitative agreement with experiments performed at the same laboratory (Knudsen et al 1986, Atan et al 1990). This has to be contrasted with the unfortunate situation that the $\beta_{1}$ values (for a given target) recorded at different laboratories show so strong variations that a comparison of the absolute value of $\beta_{1}$ with theory is hardly possible. 
$\beta_{1}$

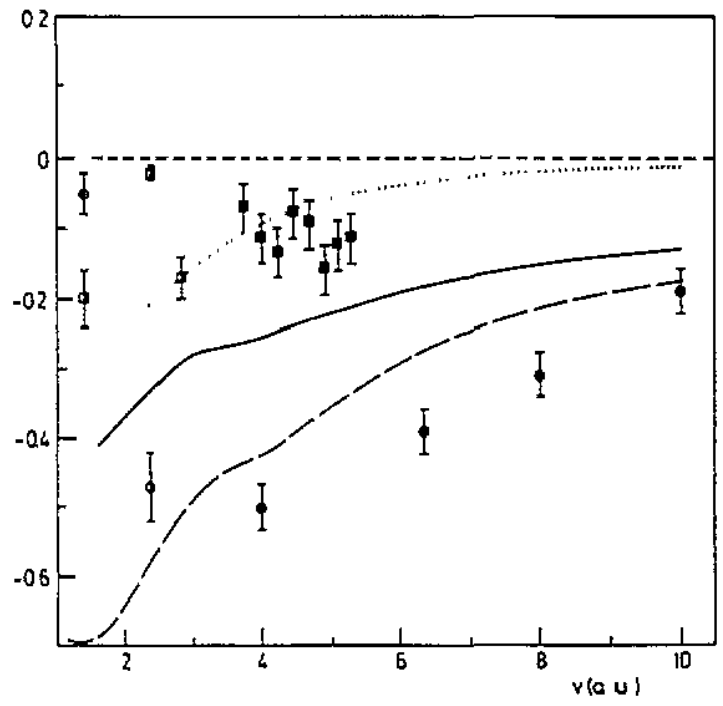

Figure 2. Cusp asymmetry parameter $\beta_{1}=B_{01} / B_{00}$ for $\mathrm{ELC}$ in $\mathrm{He}^{+}+\mathrm{He}$, Ar collisions as a function of impact velocity. Theory: SB2 (-) with SI+D1, SB2 (- $\longrightarrow$ with SI only, peaked SB2 and first Bom (- - ) with SI+DI, all for $\mathrm{He}^{+}+\mathrm{Ar}$. $\mathrm{B} 2(\ldots \ldots)$, with $\mathrm{SI}+\mathrm{DI}$ for $\mathrm{ELC}$ in $\mathrm{He}^{+}+\mathrm{He}$. Experiment (all for $\mathrm{He}^{+}+\mathrm{Ar}$ ): $\bullet$, Knudsen et al (1986) and Jensen (1986), coincidence data: E, Atan et al (1990), singles data: O, Gulyás et al (1992), coincidence data; $\square$, Gulyás et al $(1989,1992)$, singles data. $\mathrm{B}_{2}$

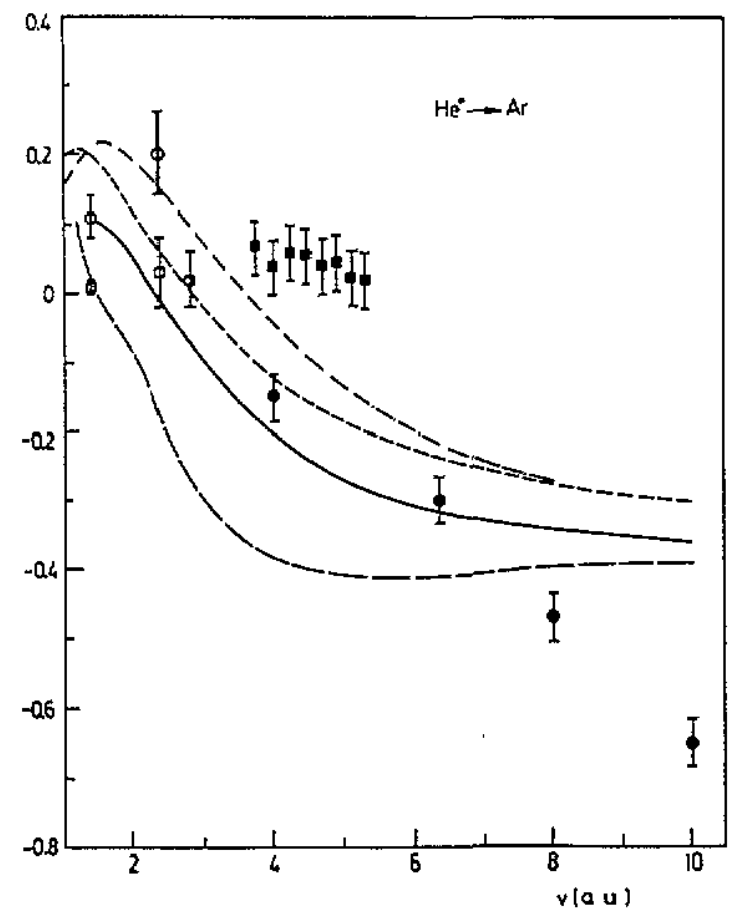

Figure 3. Cusp shape parameter $\beta_{2}=B_{02} / B_{00}$ for ELC in $\mathrm{He}^{+}+\mathrm{Ar}$ collision as a function of impact velocity. Theory: SB2 (-), peaked SB2 (- - -), first Born approximation (- - ), all theories with S1+DI contributions; SB2 ( $-\longrightarrow$ ) with SI only. Experiment: see caption of figure 2. 
The parameter $\beta_{2}=B_{02} / B_{00}$ which is a measure of the electron intensity emitted perpendicular to the beam direction (in the projectile reference frame) is plotted in figure 3 for the $\mathrm{He}^{+}+\mathrm{Ar}$ system. Theory predicts that $\beta_{2}$ is positive at small impact velocities and negative at larger $v$, a behaviour which had also been found for He targets. However, most of the experimental data are underestimated by the (unpeaked) SB2 theory, whereas the peaked SB2 and the first Born approximation seem to give better results. In view of the deficiencies of the latter theories as far as $\beta_{1}$ (and in part, $B_{00}$ ) is concerned, we consider this better agreement to be fortuitous.

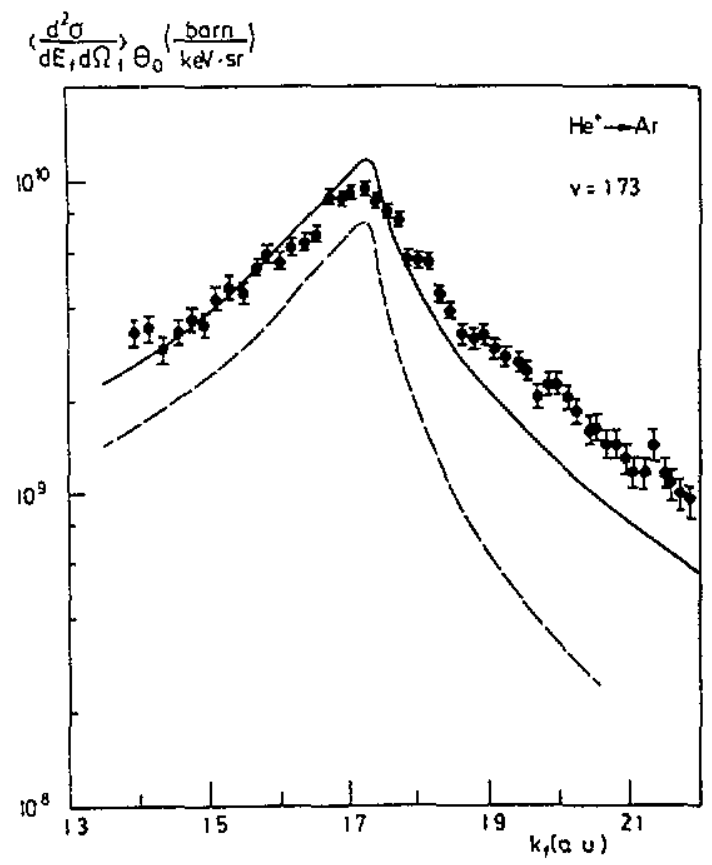

Figure 4. Forward peak for $75 \mathrm{keV} \mathrm{amu}{ }^{-1} \mathrm{He}^{+}+\mathrm{Ar}$ collision at acceptance angle $\theta_{0}=3.5^{\circ}$. Experiment: Köver et al (1989), coincidence data. The error bars do not include the absolute uncertainty of $40 \%$. Theory: $\mathrm{SB} 2(-)$ with $\mathrm{SI} \div \mathrm{DI}, \mathrm{SB} 2(---)$ with SI only.

The zero-degree electron spectrum from $75 \mathrm{keV} \mathrm{amu}{ }^{-1} \mathrm{He}^{+}+\mathrm{Ar}$ collision in the unpeaked SB2 theory is shown in figure 4. Comparison is made with experimental data recorded in coincidence with the charge state of the transmitted projectile $\left(\mathrm{He}^{2+}\right)$ in order to separate electron loss from the electron capture to continuum process which is dominating at such a low velocity (Kövér et al 1989). The SB2 theory agrees quite well with experiment, in intensity and also in the peak shape. If only the sl contribution were considered, the intensity would be too low and the asymmetry too strong as compared with the data.

As a second example, the cusp spectrum from $145 \mathrm{keV} \mathrm{amu}^{-1} \mathrm{He}^{+}$on $\mathrm{Ar}$ (where the contribution from ELC to the total yield has increased to 65\%) is given in figure 5. For this system, new high-precision coincidence data are available (Gulyás et al 1992) at a smaller acceptance angle $\left(\theta_{0}=2.5^{\circ}\right)$. We find a very good agreement between the SB2 results and the experimental data for the peak shape. Unfortunately, the data are only given on a relative scale, but from figure 1 we conjecture that the agreement in absolute value 


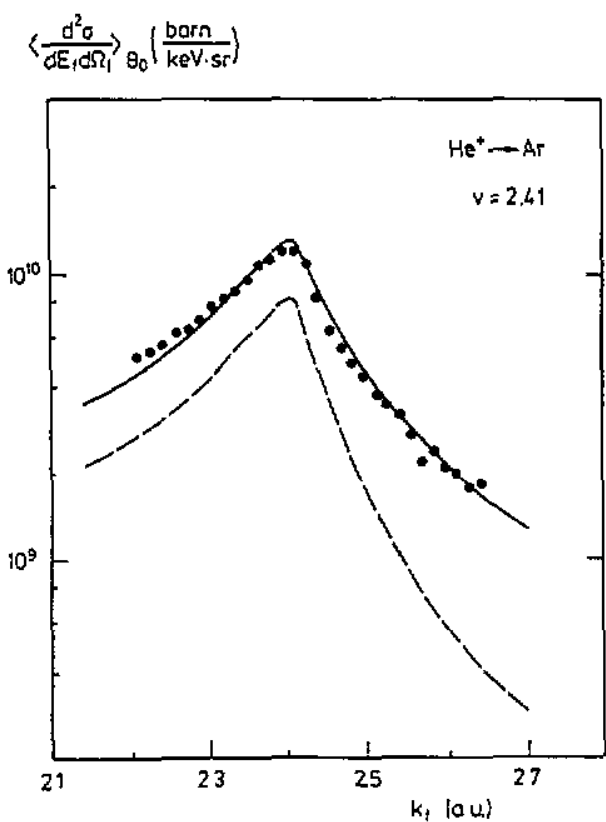

Figure 5. Forward peak for $145 \mathrm{keV} \mathrm{amu}{ }^{-1} \mathrm{He}^{+}+\mathrm{Ar}$ collision at acceptance angle $\theta_{0}=2.5^{\circ}$. Experiment: Gulyas et al (1992), coincidence data. Experiment is normalized to theory. Theory: SB2 (-) with SI+DI, SB2 (- - $\rightarrow$ with SI only.

should be within $30 \%$. As far as the shape parameters $\beta_{1}$ and $\beta_{2}$ are concerned there is, however, a considerable difference between theory and experiment for this spectrum (cf figures 2 and 3) although the general cusp shape is so well reproduced. This casts some doubt on the significance of a $B_{n l}$ expansion since obviously these parameters are extremely sensitive to small features on the top or the wings of the cusp which possibly are just caused by experimental statistics. It could therefore well be that the large scattering of the $\beta_{1}$ parameters as a function of $v$ (figure 2 ) is an artefact which should not be related to large differences in the real shape of the cusp spectrum.

\section{Electron loss at larger emission angles}

When the emission angle of the electron in the loss peak region increases, so does its energy in the projectile reference frame. This implies that the strong target potential will increasingly influence not only the intermediate states, but also the final state of the electron. Hence, a proper theory for electron emission at arbitrary angles should include the effect of both potentials $\left(V_{\mathrm{P}}\right.$ and $\left.V_{\mathrm{T}}\right)$ in the final state. This consideration leads to the well known continuum distorted wave $(\mathrm{CDW})$ theory. While the conventionally used first-order approach of this theory in general accounts reasonably well for total electron intensities (see e.g. Wang et al 1992), it may fail when one of the two fields is strongly dominating, in particular in the case of the cusp shape parameters (Crothers and McCann 1987).

We are suggesting an alternative approach which consists of a hierarchy of closely related theories which are successively applicable when the electron emission angle is increased. For small angles, when the electron energies are close to the continuum threshold of the projectile (in the cusp region), we have shown that the strong potential second Born theory 


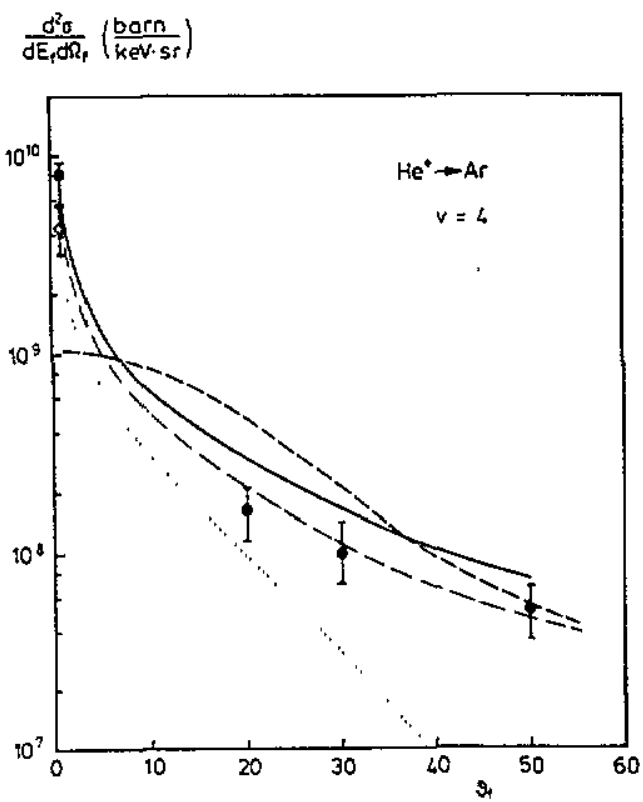

Figure 6. Doubly differential cross section at $v=k_{f}=4$ au for electron loss in $\mathrm{He}^{+}+\mathrm{Ar}$ collision as a function of electron angle $\vartheta_{f}$. Theory: SB2 (-), peaked SB2 $(-,-)$, EIA (- - ) , all theories with sI only. First Born DI $(\cdots \cdots)$. Experiment: DuBois and Marson (1990), coincidence data; $\square$, Atan et al (1990), singles data; $\diamond$, Knudsen et al (1986) and Jensen (1986), coincidence data.

is appropriate. This theory treats the intermediate electronic states as target eigenstates and the final state as a projectile eigenstate. From the SB2, the 'impulse approximation' of Hartley and Walters (1987) is readily derived by means of a peaking approximation. Although in this theory the final state is still a projectile eigenstate, the intermediate states adopt over a large region of momenta $\boldsymbol{q}^{\prime}$ the properties of a final target scattering state (with the proper momentum $k_{f}$ ). This CDW-type behaviour results from the full peaking approximation together with the on-shell prescription for the scattering amplitude. Hence, the peaked SB2 is expected to be superior to the (unpeaked) SB2 at those angles where the influence of both nuclear fields on the ejected electron is comparable. In tum, from the peaked SB2 theory, the electron impact approximation (EIA) can be formally derived. This theory has been used to describe electron loss at backward emission angles (Kuzel et al 1992) and is obtained from (2.9) by replacing the final projectile eigenstate with a plane wave, i.e.

$$
\frac{\mathrm{d}^{2} \sigma^{\mathrm{EIA}}}{\mathrm{d} E_{f} \mathrm{~d} \Omega_{f}}=\frac{k_{f}}{v} \int \mathrm{d} q\left|\varphi_{f}^{\mathrm{P}}(q)\right|^{2}\left|f\left(q+v, k_{f}\right)\right|^{2} \delta\left(E_{f}-\varepsilon_{i}^{\mathrm{P}}-v^{2} / 2-q v\right)
$$

for the singly inelastic part. This replacement is equivalent to the choice of a final target scattering eigenstate, and effectively corresponds to a change of the boundary conditions. We recall that the EIA is a first-order theory for rearrangement, and hence is appropriate at large emission angles where the target field has the dominant influence on the ejected electron.

The validity regime of the three theories is demonstrated in figure 6 , where the doubly differential electron loss cross section at the cusp energy $\left(k_{f}=v\right)$ is plotted as a function 
of ejection angle. The SI and DI contributions are shown separately in order to display the strong decrease of the first-order DI theory with angle. Clearly, for angles $\vartheta_{f} \gtrsim 30^{\circ}$, the second-order DI contribution can no longer be neglected, but will add to the loss cross section.

Comparison is made with the large-angle data of DuBois and Manson (1990). Also shown are data points at $\vartheta_{f}=1^{\circ}$, which are-in the case of the Knudsen et al (1986) data-obtained from the tabulated $B_{0 !}$ values shown in figures 1-3 with the help of the series (4.2) truncated to $n=0$ and $l \leqslant 2$, or-in the case of the Atan et al (1990) dataextracted from an extrapolated value of $B_{00}$ from figure 1 with the help of the relation (4.4a). The two procedures give very similar results, their difference being $7 \%$ for Knudsen's and $2 \%$ for Atan's data.

From the comparison with experiment it is confirmed that the $\mathrm{SB} 2$ theory is reasonable for $\vartheta_{f} \lesssim 50^{\circ}$ for $400 \mathrm{keV} \mathrm{amu}-1 \mathrm{He}^{+}$on Ar in the loss peak region. The EIA theory (which is calculated from (5.1) without additional peaking approximations) is applicable for $\vartheta_{f} \gtrsim 40^{\circ}$. ElA fails for very small $\vartheta_{f}$ since it does not account for the cusp phenomenon. The best description in the intermediate region, $20^{\circ} \lesssim \vartheta_{f} \lesssim 40^{\circ}$, where the two-centre effects on the final state are most important (for $k_{f}=v$ ), is indeed provided by the peaked SB2 theory.

\section{Conclusion}

We have derived the strong potential second Born theory for electrons emitted from light projectiles in collision with heavy targets in the loss peak region at forward angles. In this theory, the Born series is summed to infinite order in the target field, thus correcting for serious deficiencies of the first- and second-order terms of this series. We have applied the SB2 to the calculation of the elastic contribution to electron loss where the target is left in its ground state. Upon adding the first-order inelastic contribution, we have found that SB2 gives a good accord of intensity and shape of the forward peak, thus improving on the 'impulse approximation' of Hartley and Walters. For electrons in the loss peak region, $\mathrm{SB} 2$ is valid for emission angles up to $\sim 50^{\circ}$. On the other hand, the impulse approximation' is superior for $\vartheta_{f} \gtrsim 20^{\circ}$. For large emission angles $\left(\vartheta_{f} \gtrsim 40^{\circ}\right)$ the electron impact approximation should be used. We have shown that these three theories are closely related by means of successive approximations which progressively enhance the influence of the target field on the emitted electron. Thus we have finally found a prescription of electron loss which is valid in the complete range of electron energies and emission angles for asymmetric collision systems.

\section{Acknowledgments}

I should like to thank $M$ W Lucas and W Oswald for many helpful discussions. I am also indebted to L Gulyás, $\mathrm{H}$ Knudsen and M W Lucas for the communication of unpublished experimental data. Support from the GSI Darmstadt is gratefully acknowledged.

\section{Appendix}

We are giving details for the evaluation of the strong potential second Born cross section (2.15). 
For the calculation of the elastic scattering amplitude $f_{\mathrm{e}}(K, \theta)$ we have determined the partial wave phaseshifts from the numerical solution of the Schrödinger equation for the radial wavefunction

$$
\left(\frac{\mathrm{d}^{2}}{\mathrm{~d} r^{2}}+K^{2}-\frac{l(l+1)}{r^{2}}-2 V_{\mathrm{T}}(r)\right) R_{l}(K, r)=0
$$

The target potential is approximated by an analytical fit to the static Hartree-Fock potential, to which a polarization field is added

$$
\begin{aligned}
& V_{\mathrm{T}}(r)=-\frac{Z_{\mathrm{T}}}{r} \sum_{i}\left(a_{i} \mathrm{e}^{-b_{i} r}+\alpha_{i} r \mathrm{e}^{-\beta_{i} r}\right)-\frac{\alpha r^{2}}{2\left(r^{2}+d^{2}\right)^{3}} \\
& d=\frac{3}{8 \Delta} \begin{cases}k_{0} & K \leqslant k_{0} \\
K & K>k_{0}\end{cases}
\end{aligned}
$$

where for Ar, the parameters $a_{i}, b_{i}, \alpha_{i}, \beta_{i}$ are taken from the tables of Strand and Bonham (1964) while $\alpha=13.333, \Delta=0.58$ and $k_{0}=2$ as in previous work. The phaseshifts are considered for $l \leqslant 16$ and are calculated prior to the evaluation of the cross section $(2,15)$ on a grid of mesh points $K_{i}$ (typically, $i_{\max } \sim 20$ ). Subsequently for each momentum $K_{i}$, $f_{e}\left(K_{i}, \theta\right)$ is evaluated at the actual value of the scattering angle $\theta$, and $f_{e}(K, \theta)$ is found with the help of an interpolation routine. This method differs from the one used by Hartley and Walters (1987) who interpolate the tabulated experimental cross sections for elastic electron scattering. It has the advantage of being applicable to any target, without the need of experimental data. Moreover, in the SB2 theory, it is the scattering amplitude and not the cross section $\left|f_{\mathrm{e}}(K, \Theta)\right|^{2}$ which enters into the calculation.

The singularities in the integrand of (2.15) at $k^{\prime}=0$ and $x=-k^{\prime} / 2 \kappa_{f}$ deserve a special treatment, the latter leading to a branch point at $k^{\prime}=2 \kappa_{f}$. Hence, the $k^{\prime}$ integral is split at $k^{\prime}=2 \kappa_{f}$ and the pole is treated analytically

$$
\int_{\delta}^{k_{\max }} \frac{\mathrm{d} k^{\prime}}{k^{\prime 1-i \eta}} F\left(k^{\prime}\right)=\left(\int_{\delta}^{2 k_{f}-\varepsilon} \mathrm{d} k^{\prime}+\int_{2 x_{f}+\varepsilon}^{k_{\max }} \mathrm{d} k^{\prime}\right) \frac{1}{k^{\prime 1-i \eta}}\left[F\left(k^{\prime}\right)-F(0)\right]+F(0) \frac{1}{\mathrm{i} \eta}\left(k_{\max }\right)^{i \eta}
$$

with $\delta, \varepsilon \rightarrow 0$ and $k_{\max } \rightarrow \infty$. In addition, a logarithmic variable substitution, $y=\ln k^{\prime}$, should be made in both integrals. For $k^{\prime}>2 \kappa_{f}$, no singularity is present in the $x$ integral. In this case, the function $F\left(k^{\prime}\right)$ is given by

$$
\begin{aligned}
& F\left(k^{\prime}\right)=\int_{-1}^{1} \mathrm{~d} x\left(k^{\prime}+2 \kappa_{f} x-\mathrm{i} \varepsilon\right)^{-\mathrm{i} \eta-1} W\left(k^{\prime}, x\right) \quad k^{\prime}>2 \kappa_{f} \\
& W\left(k^{\prime}, x\right)=\frac{A}{\left(A^{2}-B^{2}\right)^{3 / 2}} f_{\mathrm{e}}(K, \theta) .
\end{aligned}
$$

In the region $k^{\prime}<2 \kappa_{f}$, the singularity has to be treated analytically, such that $F\left(k^{\prime}\right)$ is calculated from

$$
\begin{aligned}
F\left(k^{\prime}\right)=\left(\int_{-1}^{\bar{x}-\varepsilon} \mathrm{d} x+\int_{\bar{x}+\varepsilon}^{1} \mathrm{~d} x\right)\left(k^{\prime}+2 \kappa_{f} x-\mathrm{i} \varepsilon\right)^{-\mathrm{i} \eta-1}\left[W\left(k^{\prime}, x\right)-W\left(k^{\prime}, \bar{x}\right)\right] & \\
& +W\left(k^{\prime}, \bar{x}\right) \frac{1}{2 \mathrm{i} Z_{\mathrm{P}}}\left[\left(k^{\prime}-2 \kappa_{f}-\mathrm{i} \varepsilon\right)^{-\mathrm{i} \eta}-\left(k^{\prime}+2 \kappa_{f}-\mathrm{i} \varepsilon\right)^{-\mathrm{i} \eta}\right] \quad k^{\prime}<2 \kappa_{f}
\end{aligned}
$$


with $\bar{x}=-k^{\prime} / 2 k_{f}<0$. Again, it is useful to make logarithmic variable substitutions $y=\ln (\bar{x}-x)$ for $x<\bar{x}$ and $y=\ln (x-\bar{x})$ for $x>\bar{x}$. We have restricted the integration over $\varphi_{q^{\prime}}$ to the interval $(0, \pi)$ and multiplied the cross section by 2 . This is quite accurate since $-C_{0} / B$ from (2.13) is mostly below -1 where $\sin \varphi_{q^{\prime}}$ does not occur. Even then, the evaluation of the doubly differential SB2 loss cross section at fixed $k_{f}$ and $\vartheta_{f} \neq 0$ takes about $6 \mathrm{~h}$ on a Sparc workstation (or $3 \mathrm{~h}$ on a Cyber 2000). The numerical accuracy is about $10 \%$. Additional inaccuracies of our theoretical results may arise from the on-shell approximation (2.12), and to a minor extent from deficiencies of the target potential $V_{\mathrm{T}}$ entering into the calculation of the phaseshifts.

\section{References}

Atan H, Steckelmacher W and Lucas M W 1990 J. Phys. B: At. Mol. Opt. Phys. 232579

Bates D R and Griffing G 1954 Proc. Phys. Soc. A 67663

Briggs J S and Drepper F 1978 J. Phys. B: At, Mol. Phys. 114033

Crothers D F S and McCann J F 1987 J. Phys. B: At. Mol. Phys. 20 L19

Day M H 1980 J. Phys. Bi At. Mol. Phys, 13 L65

1981 J. Phys. B: At. Mol. Phys. 14231

Drepper F and Briggs J S 1976 J. Phys. B: At. Mol. Phys. 92063

DuBois R D and Manson S T 1990 Phys. Rev. A 421222

Gulyás L, Sarknđi L, Pálinkás J, Kövér Á, Vajnaj T, Szabó Gy, Végh J, Berényi D and Elston S B 1992 Phys. Rev. A 454535

Gulyás L, Szabó Gy, Kövér Á, Berényi D, Heil O and Groeneveld K O 1989 Phys. Rev. A 394414

Hartley H M and Walters H R J 1987 J. Phys. B; At. Mol. Phys. 203811

Jakubassa D H 1980 J. Phys. B: At. Mol. Phys. 132099

Jakubassa-Amundsen D H 1990 J. Phys. B; At. Mol. Opt. Phys. 233335

Jensen K E 1986 Thesis University of Aarhus

Kahle M 1991 Diploma Thesis University of Munich

Knudsen H, Andersen L H and Jensen K E 1986 Int. Symp. on Physics of lonized Gases (Sibenik, Yugosiavia) Progress Report

Kövêr A, Sarkadi L, Pálinkás J, Berényi D, Szabó Gy, Vajnai T, Heil O, Groeneveld K O, Gibbons I and Sellin I A 1989 J. Phys. B: At. Mol. Opt. Phys. 221595

Kuzel M, Heil O, Maer R, Lucas MW, Jakubassa-Amundsen D H, Farmery B W and Groeneveld K O 1992 J. Phys. B: At. Mol. Opt. Phys. 251839

Man K F, Steckelmacher W and Lucas M W 1986 J. Phys. B: At. Mol. Phys. 19401

Meckbach W, Nemirovsky I B and Garibotti C R 1981 Phys, Rev. A 241793

Montenegro E C and Meyerhof W E 1991 Phys, Rev. A 432289

Strand T G and Bonham R A 1964 J. Chem. Phys. 401686

Taulbjerg K, Barrachina RO and Macek I H 1990 Phys. Rev. A 41207

Walters H R J 1975 J. Phys. B: At. Mol. Phys. 8 L54

Wang J, Reinhold C O and Burgdörfer J 1992 Phys. Rev. A 454507 